\title{
Laboreal
}

Volume $13 \mathrm{~N}^{\circ} 1$ | 2017

Varia

\section{La aptitud profesional según J.-M. Lahy}

A aptidão profissional segundo J.-M. Lahy

L'aptitude professionnelle selon J.-M. Lahy

The occupational skill according to J.-M. Lahy

\section{Marcel Turbiaux}

Traductor. Cecilia De la Garza y Mario Poy

\section{(2) OpenEdition}

\section{Journals}

Edición electrónica

URL: http://journals.openedition.org/laboreal/1994

DOI: $10.4000 /$ laboreal. 1994

ISSN: 1646-5237

Editor

Universidade do Porto

\section{Referencia electrónica}

Marcel Turbiaux, «La aptitud profesional según J.-M. Lahy », Laboreal [En línea], Volume 13 Nº 1 | 2017 Publicado el 01 julio 2017, consultado el 23 septiembre 2020. URL : http://journals.openedition.org/ laboreal/1994; DOI : https://doi.org/10.4000/laboreal.1994

Este documento fue generado automáticamente el 23 septiembre 2020

\section{c) (i) (8)}

Laboreal está licenciado com uma Licença Creative Commons - Atribuição-NãoComercial 4.0 Internacional. 


\title{
La aptitud profesional según J.-M. Lahy
}

\author{
A aptidão profissional segundo J.-M. Lahy \\ L'aptitude professionnelle selon J.-M. Lahy \\ The occupational skill according to J.-M. Lahy
}

Marcel Turbiaux

Tradución : Cecilia De la Garza y Mario Poy

\section{REFERENCIA}

Comentário do texto de J.-M. Lahy, « Qu'est-ce qu'une aptitude professionnelle ? - 0 que é uma aptidão profissional?» $3^{\mathrm{a}}$ conferência internacional de psicotécnica, Milan, 2-4 Outubro 1922.

\section{NOTA DEL EDITOR}

http://dx.doi.org/10.15667/laborealxiii0117mtes

Manuscrito recibido en: Diciembre/2016

Aceptado tras peritaje: Abril/2017

La aptitud, un concepto clave de la orientación profesional y de la selección profesional, ha sido objeto de numerosos debates en el marco del nacimiento y desarrollo de la psicotécnica. Desde 1919, Frédéric Özil (1879-1967), creador de la Ecole universelle en 1907, funda la revista, L'orientation professionnelle. En 1920, Julien Fontègne (1869-1945) elabora un "índice bibliográfico sobre el tema la orientación profesional de los jóvenes ", que ya cuenta con 333 títulos que dan testimonio de su importancia. El comentario del texto de J.-M Lahy (1872-1942), "¿Qué es una aptitud profesional?", representa la oportunidad de retomar los debates que han atravesado a la orientación profesional en la primera mitad del siglo XX. 
1. Cuando publica este texto, Lahy es jefe de trabajos prácticos en el laboratorio de psicología experimental de la École pratique des hautes études, fundado en 1898, en el asilo de Villejuif, por Edouard Toulouse, médico jefe (1865-1947), al que se incorporó como estudiante en 1901. Toulouse había llevado adelante, en 1895, una "investigación médico-psicológica acerca de las relaciones entre la superioridad intelectual y neuropatía". Su primer sujeto fue Emile Zola (1840-1902), a quien estudia mediante la observación directa, el examen clínico y el uso de técnicas experimentales (mental test). La presentación de su ensayo en la Sociedad médico-psicológica en 1897, concluía recomendando el método que había utilizado para estudiar a Zola, pero no solo de la superioridad intelectual sino, también, acerca de las "condiciones de superioridad en todas las profesiones, incluyendo las ocupaciones manuales". Con este objetivo, en 1900, desarrolla en el Ministerio de Comercio un proyecto de un "laboratorio de investigaciones biológicas y sociales", cuya finalidad es, entre otras cosas, la de organizar la selección profesional. Este proyecto no fue ejecutado, pero el laboratorio de psicología fisiológica recibió una subvención y Toulouse confiará a J.-M. Lahy el estudio de superioridad profesional en las ocupaciones. Este último, en 1903, se centrará en el estudio de una nueva profesión, la de la mecanógrafa, buscando, de acuerdo al método recomendado por Toulouse, las condiciones de superioridad profesional en esta ocupación y, en 1908, en los conductores de tranvías [1] y la psicofisiología del soldado artillero en 1916.

En 1913, haciendo un balance de su investigación sobre las mecanógrafas, utiliza la palabra "aptitud, que la caracteriza por una buena memoria de frases concretas, una tendencia a la equivalencia de las dos manos, una afinada sensibilidad táctil y muscular, una atención sostenida"(p. 833). En 1922, a propósito de la orientación profesional a la cual se le consagró en Ginebra, en 1920, el primer congreso de la psicotécnica “aplicada a la orientación profesional" a fin de intercambiar ideas sobre el tema de las "aptitudes innatas y las aptitudes adquiridas ", por iniciativa del Instituto Jean-Jacques Rousseau, el autor va a definir lo que él entiende por aptitudes como: "las disposiciones naturales que se reconocen por respuestas motrices o mentales medibles frente a ciertas excitaciones dadas " y, más específicamente, a las aptitudes profesionales como las "disposiciones naturales así definidas cuyos valores son del orden de los valores típicos reconocidos en los trabajadores de élite."

Edouard Toulouse, en 1903 [²], en Le Journal profetizaba que: "Llegará el día en el cual el concurso consistirá en un examen médico-psicológico, en el que se mostrarán las habilidades particulares que serán valoradas, y ningún padre va a empujar a su hijo a una actividad comercial o profesión sin haberlo hecho previamente examinar" y retomará esta cuestión en "Le petit journal" el 24 de agosto de 1921 ("La vocation "), sosteniendo que "lo que importa es la selección de cada individuo de acuerdo a sus aptitudes verdaderas [...] la aptitud real se encuentra en un pequeño número de cualidades fisiológicas que posibilitan llevar a cabo en forma rápida y segura los actos de una profesión dada". Y esto "parece ser cierto tanto para el obrero como para el ingeniero que dirige una fábrica", y en "L'ère nouvelle", del 27 de febrero de 1921 ("L’orientation professionnelle"), afirma que: "sólo la ciencia es capaz de determinar mediante pruebas (...) rápidas las relaciones entre aptitudes y ciertos modos de reacción durante el transcurso de experiencias elegidas convenientemente" anunciando una conferencia de (su) colaborador Lahy quien es, desde hace ya mucho tiempo, especialista en el estudio de la orientación profesional ". 
Lahy (1922) desarrolla la idea de " buscar ante todo las aptitudes características de una profesión y clasificarlas en orden de importancia para saber cuáles dan la superioridad, es preciso conocer todas las aptitudes necesarias para realizar acabadamente la mayoría de los trabajos realizados por el hombre y determinar para cada aptitud su orden de magnitud en los diferentes individuos".

En su intervención en el congreso de Milán, explícitamente dedicado a la definición del concepto de aptitud, Lahy retoma la definición, que había dado en Le bulletin médical de ese año, aplicable a la selección profesional y, también la orientación profesional. De hecho, la intervención de Lahy en el Congreso de Milán es un alegato en favor de su concepción de la aptitud profesional y de su determinación, que defenderá y precisará en varios escritos que había aplicado, y que aplicará en su investigación y en su crítica del "Sistema Taylorista" [3].

2. La exposición de Lahy es discutida profundamente. Las discusiones revelan las vacilaciones, en ese momento, acerca de la teoría de las aptitudes. En tal sentido, Claparède escribió en 1923: "El tema de las aptitudes todavía está envuelto por la oscuridad."

L. Remy compara "aptitud" y "hábito": "La aptitud es una disposición natural para llevar a cabo ciertos actos, que depende de una disposición innata; el hábito es una disposición adquirida para realizar un gran número de actos similares. La aptitud, de la misma manera que el hábito, aumenta la cantidad de rendimiento, en cambio solo la aptitud aumenta la calidad. La perfectibilidad depende de las facultades intelectuales. Por lo tanto, hay tantos grupos de aptitudes como de facultades intelectuales, es decir que existen aptitudes de los centros intelectuales superiores, centros de memoria y de aptitudes sensoriales. Estas aptitudes se pueden medir y por lo tanto medir la inteligencia de los niños. La escuela debe contribuir entonces a la organización de la orientación profesional, proporcionando las informaciones recogidas por la psicología y por la pedagogía experimental durante los años escolares".

Según el autor, "las aptitudes intelectuales superiores tienen una importancia considerable en las aptitudes de los diferentes oficios ya que, cualquier acto, cualquiera que sea su naturaleza, requiere de la intervención de los centros intelectuales superiores que regulan la ejecución de movimientos [...] Los mejores órganos sensoriales no siempre se corresponden con los mejores centros intelectuales superiores. Por lo tanto, debemos tener en cuenta no sólo la cantidad, sino también la calidad de las excitaciones recogidas en el mundo exterior".

Eugenio Rignano (1870-1930) piensa que la psicotécnica se ha ocupado hasta el presente exclusivamente de las aptitudes intelectuales $y$ ha descuidado las disposiciones afectivas.

Hugo Heinis (1883-1967), del gabinete de orientación experimental del Instituto JeanJacques Rousseau creado en 1918, a propósito del concepto de inteligencia general, es de la opinión de que no hay uno, sino varios tipos de inteligencia. Admite que la inteligencia general es la capacidad de adaptarse a las situaciones nuevas, si se la considera en un sentido amplio. En todos sus experimentos, que sobrepasan al millar, nunca encontró un sujeto que sea bueno en todos los ámbitos. Él cree que "el hombre no tiene dicha inteligencia general; lo que tenemos es una inteligencia parcial. Por lo tanto desde el punto de vista de la psicología aplicada [...] sería mejor ubicar a esta inteligencia parcial de acuerdo a cada oficio, que abordar problemas demasiado generales". 
Arthur Guillaume Christiaens (1860-1937), director de Office intercommunal d'orientation professionnelle de Bruxelles, abierto desde 1910, considera, por el contrario, la definición de la aptitud de Lahy aceptable: "La aptitud es el resultado de la influencia de los estímulos ambientales sobre los elementos nerviosos, cuya calidad depende de la herencia. Dado que las variadas existencias de la vida laboral no pueden ser reproducidas a voluntad, en el momento del examen, ha sido necesario recurrir a los tests para reemplazarlas".

Edouard Claparède (1873-1940), fundador en 1912 del Instituto Jean-Jacques Rousseau, y presidente de la Association internationale de psychotechnique, de la cual Lahy fue el secretario general permanente, estima que no es posible separar, en la práctica, la aptitud adquirida y la aptitud innata subrayando la necesidad de ponerse de acuerdo acerca de la elección de los tests.

Gerard Anton Marie van Wayenburg, profesor de psicología infantil en la universidad y director del laboratorio de psicotécnica de Ámsterdam, propone su definición de la aptitud: la aptitud es un complejo de actos fisiológicos y psicológicos coordinados por un objetivo. Algunas habilidades se pueden desarrollar, más o menos tardíamente; y es por ello que es necesario saber qué aptitudes se pueden desarrollar más tarde.

Otto Lipmann [4] (1880-1933), Director del Instituto de Psicología Aplicada de Berlín y director del Zeitschriftfürangewandte Psychologie, considera que, en la cuestión de la inteligencia general se debería de tomar en cuenta dos cuestiones separadas: ¿Existe una inteligencia general? ¿Cómo se puede reconocer la inteligencia general? Y da su respuesta a esas dos preguntas:

La inteligencia general no existe, en el sentido en que una persona que entiende bien y rápidamente las preguntas intelectuales es también inteligente cuando se trata de adaptarlas a las cosas o a las personas, o bien de aplicarlas a los problemas técnicos. Son dos tipos de inteligencia diferentes, pero esta diferencia tiene un significado más teórico que practico, ya que en las diversas profesiones que se examinan para la selección de una profesión, no se pide al principio más que una sola de esas inteligencias, que se le calcula como inteligencia general.

Para establecer el grado de inteligencia general, una prueba (test) no basta, sino al menos une serie de pruebas, del tipo de aquellas de Binet-Simon o como las del Army test américain [5]. El autor pone en relieve que uno de los mejores medios para establecer esta inteligencia es la observación, la cual permite no solo observar un mayor número de características en la persona examinada, sino también une idea más precisa de su personalidad; la observación, que permite no solo estudiar su porte natural, sino también ver como ésta se comporta ante una prueba. La orientación profesional puede apoyarse en esas observaciones, pero la selección debe basarse en particularidades más detalladas.

Claparède agrega otro punto al de Lipmann: ¿Cuál es la influencia de la inteligencia general sobre la profesión? Se refiere a los estudios de Hugo Münsterberg (1863-1916) y otros que aplicaron tests a estudiantes y estos sobrepasaron los sujetos de diversas categorías, lo que comprueba el valor de la inteligencia general. En consecuencia, convendría establecer para cuales profesiones la inteligencia general es más útil y aquellas para las cuales lo es menos.

Enfin, A. J. Petitpierre, director de la escuela de los oficios de Lausanne, juzga el término de inteligencia general demasiado vago. Considera que las aptitudes profesionales deben ser definidas según las profesiones, las cuales separa en tres 
grupos: las profesiones para las cuales las aptitudes intelectuales prevalecen, las profesiones para las cuales las aptitudes manuales prevalecen, las profesiones para las cuales un equilibrio entre les dos tipos de aptitudes es necesario.

3. Sin embargo, en 1931, Franziska Baumgarten (1889-1970), nota en relación con la definición de Lahy: "Por más interesante que es esta definición, comprende la necesidad de rendimientos más allá del promedio. No se le puede considerar como susceptible de aplicaciones prácticas, ya que los rendimientos elevados solo se encuentran en una minoría en todas las profesiones. La "aptitud profesional", como toda aptitud, no puede ser definida como une disposición encontrándose en un nivel definido elevado, pero como una disposición pudiendo variar por grado (grande, mediana, pequeña)" (p. 17-18).

Maurice Reuchlin (1920-2015), es aún más duro. Al criticar (1954, p. 374) "la extraordinaria vitalidad de (la) asimilación falsa del resultado al test con una aptitud congénita", el autor escribe: "Se encuentra esta asimilación [...] en la obra de J.-M. Lahy [...] Lahy está por encerrarse en la tautología [...] ya que después de haber definido las aptitudes como disposiciones naturales que se reconocen por las respuestas motrices o mentales -medibles- según excitaciones definidas, el define el test [...] como una "prueba de examen psicológico permitiendo apreciar tal aptitud mediante los resultados provistos por el sujeto". Tal definición puede crear una ambigüedad ya que la aptitud está presente tanto como "disposición natural" y "reconocible por respuestas motrices o mentales". ¿Qué medio tenemos para identificar la disposición natural en la respuestas medidas?"

Mientras tanto, Pierre Naville (1904-1993) criticaba la definición de la aptitud como “disposición natural" y definía la aptitud profesional como "un producto dialectico, el resultado de interacciones entre el medio social económico [...] y los organismos individuales, entre las formas biológicas y sociales de grupos humanos. Es una forma especial de adaptación". Piéron (1945), para quien la aptitud era la "condición congénita de una cierta modalidad de eficiencia", le contestara, precisando la distinción que según él, debe hacerse ente aptitud, "disposición innata" y capacidad, "resultado adquirido por el ejercicio".

Así, como lo resume Antoine Léon (1921-1998), "pasamos de una especie de fatalismo de la herencia sustentada por la noción de disposición natural a una especie de fatalismo del medio, relacionado a la idea de diferencias culturales irreversibles" (1980, p. 168).

Hoy en día, el deslizamiento semántico de la aptitud a la competencia no debe permitir que olvidemos que el análisis de esta tensión sigue siendo el desafió mayor del trabajo de los investigadores y profesionales de la orientación vocacional.

\section{BIBLIOGRAFÍA}

Baumgarten, F. (1931). Les examens d'aptitude professionnelle, Paris : Dunod. 
Binet, A. (1910). Avant-propos : bilan de l'année 1910, L'année psychologique, 17, 1, V-XI.

Claparède, E. (1923). Comment diagnostiquer les aptitudes chez les écoliers. Paris : Flammarion.

Fontègne, J. (1920). Répertoire bibliographique sur la question d'orientation professionnelle de la jeunesse, Bulletin de l'Association française pour la lutte contre le chômage, 38-39, 45-62.

Lahy, J.-M. (1913). Les conditions psycho-physiologiques de l'aptitude au travail dactylographique, Journal de physiologie et de pathologie générale, 4, 826-834.

Lahy, J.-M. (1922). La psychologie expérimentale base de l'orientation professionnelle, Le bulletin médical, 24-27 mai, 438-441.

Lahy, J.-M. (1922). La sélection psycho-physiologique des travailleurs, Bulletin mensuel de la Ligue d'hygiène mentale, 1-2, 24-27.

Léon, A. (1980). Introduction à l'histoire des faits éducatifs. Paris : Presses Universitaires de France.

Lipmann, O. (1912). The school in the service of vocational study, British journal or psychology, XII, 4, 337-351.

Naville, P. (1945). Théorie de l'orientation professionnelle. Paris : Gallimard.

Piéron, H. (1912). Le domaine psychologique, L'année psychologique, 1, 1-26. https://doi.org/ 10.3406/psy.1912.3872

Piéron, H. (1914). Une œuvre psychologique de guerre : l'examen des aviateurs, L'année psychologique, 21, 237-252. https://doi.org/10.3406/psy.1914.8022

Piéron, H. (1945). La notion d'aptitude à propos d'une théorie de l'orientation professionnelle. Bulletin de l'Institut National d'Orientation Professionnelle,11-12, 169-175.

Reuchlin, M. (1954). Le problème théorique de la connaissance des aptitudes, Traité de psychologie applique, t. III, 371-394. Paris : Presses Universitaires de France.

Yoakum, C. S. \& Yerkes, R. M. (1920). Army mental tests. New York: Henry Holt et Cie. https:// doi.org/10.1037/11054-000

\section{NOTAS}

1. Piéron (1912, p. 15) criticó rápidamente esta noción de superioridad, "concepto de valor" (que) no debe tener lugar en la ciencia [...] Es nuestro estatus social el que nos lleva a apreciar más tal o cual aptitud".

2. Del mismo modo, Alfred Binet (1910) esperaba publicar en 1912 un estudio "sobre la diversidad de las aptitudes de los niños", subrayando "el inmenso interés que tendría comunicar a cada uno su valor, y la profesión a la cual la naturaleza le ha reservado un destino ». Su muerte en 1911, se lo impidió.

3. En el próximo número de Laboreal aparecerá la introducción comentada de J.-M. Lahy de su libro sobre el taylorismo, publicado en 1916.

4. Lipmann (1912) ya se había expresado largamente sobre la noción de aptitud.

5. Tests utilizados por la armada americana para el reclutamiento, en 1917-1918 (Yoakum, C. S. \& Yerkes, R. M. 1920). 


\section{AUTORES}

\section{MARCEL TURBIAUX}

Groupe de Recherche et d'Etude sur l'Histoire du Travail et de l'Orientation (GRESHTO)

Grupo de Pesquisa e de Estudo da História do Trabalho e da Orientação (GRESHTO)

Centre de Recherche sur le Travail et le Développement (CRTD)

Centro de Pesquisa sobre o Trabalho e o Desenvolvimento

Conservatoire National des Arts et Métiers (CNAM)

Conservatório Nacional das Artes e Ofícios (CNAM)

41, Rue Gay Lussac 75005, Paris, France

mturbiau@club-internet.fr 